\title{
Recent Applications of Sub-20meV Monochromated STEM-EELS: from Phonon to Core Losses in Real and Momentum Spaces
}

Q.M. Ramasse ${ }^{1}$, F.S. Hage ${ }^{1}$, D.M. Kepaptsoglou ${ }^{1}$, P. Abellan ${ }^{1}$, J. Yates ${ }^{2}$, R.J. Nicholls ${ }^{2}$, H.C. Nerl ${ }^{3}$, V. Nicolosi ${ }^{3}$, K. Winther ${ }^{4}$, K. Thygesen ${ }^{4}$, P.Z. El Khoury ${ }^{5}$, W.P. Hess ${ }^{5}$, F. Azough ${ }^{6}$, R. Freer ${ }^{6}$

1. SuperSTEM Laboratory, STFC Daresbury Campus, Keckwick Lane, Daresbury WA4 4AD, U.K.

2. Department of Materials, University of Oxford, Parks Road, Oxford OX1 3PH, U.K.

3. CRANN \& AMBER and School of Physics, Trinity College Dublin, Dublin 2, Ireland

4. Center for Atomic-Scale Materials Design, Department of Physics, Technical University of Denmark, Fysikvej, 2800, Kgs. Lyngby, Denmark

5. Physical Sciences Division, Pacific Northwest National Laboratory, Richland, WA 99352, USA

6. School of Materials, Materials Science Centre, University of Manchester, Manchester M13 9PL, U.K.

A new generation of electron beam monochromators has recently pushed the energy resolution of (scanning) transmission electron microscopes deep into the sub $20 \mathrm{meV}$ range [1]. In addition to the obvious increase in resolution which has made exploring the phonon region of the EELS spectrum possible [1], the increased flexibility of these instruments is proving hugely advantageous for materials science investigations. The energy resolution, beam current and electron optics can be adjusted seamlessly and traded off each other as necessary within a greatly increased range, as will be illustrated on a number of systems studied using a Nion UltraSTEM100MC 'Hermes' instrument recently installed at the SuperSTEM Laboratory.

In an early application to the field of two-dimensional materials, the cubic ( $c$-BN) and hexagonal ( $h$-BN) phases of boron nitride were shown to give rise to markedly different phonon excitations in EELS [2]. A better understanding of this phonon response can be achieved by observing the dependence of the phonon peak under different optical conditions, mapping the energy of the peaks in momentum space and showing a very limited dispersion with momentum, as expected for these optical phonons. Similarly, the low loss spectrum of carbon nanotubes contains a variety of characteristic losses, such as van Hove singularities which are of great importance to understand their electronic behavior and the role of defects in their electronic structure [3]. Observing these directly both in real and momentum space down to sub-20meV energy resolution for nanotubes with increasing number of walls provides insights into the possible structural origins of these excitations.

Moving up in energy loss, the band gap of semi-conductors is known to be strongly affected by structural variations such as composition or crystalline defects. Local compositional changes in $\mathrm{Cu}(\mathrm{InGa}) \mathrm{Se}_{2}$ solar cell absorber layers lead to subtle band gap changes [4], and we show how the increased energy resolution achieved through monochromation can provide an increased precision on these nm-scale measurements. We also highlight how peaks associated with excitonic effects in $\mathrm{MoS}_{2}$ nano-flakes exhibit a clear spatial dependence at step edges and on terraces of varying thicknesses, comparing these results to theoretical calculations explicitly taking into account the electron-hole interactions.

Finally the flexibility afforded by the new generation of monochromators allows us to balance beam intensity and energy width to obtain unprecedented signal-to-noise levels at energy resolution tailored to match the intrinsic width of the observed excitation, as demonstrated in mapping localized plasmon 
resonances in Ag plasmonic nanostructures [5] or in linking the crystal field splitting changes in Ti $L_{2,3}$ core loss fine structure across an A-site deficient perovskite to observed structural variations in atomic site occupancies [6].

\section{References}

[1] O.L. Krivanek et al., Phil. Trans. Roy. Soc. 367 (2009), pp. 3683-3697; T.Miyata et al., Microscopy 5 (2014), pp. 377-382 ; O.L. Krivanek et al., Nature 514 (2014), pp. 209-212.

[2] R. Nicholls et al., Microsc. Microanal. 21 Suppl.3 (2015), pp. 1469-1470.

[3] T Pichler et al., PRL 80 (1998), 4729.

[4] D. Keller et al., Microsc. Microanal. 20 (1998), pp. 1246-1253.

[5] P.Z. El Khoury et al., Submitted (2016).

[6] SuperSTEM is funded by the UK Engineering and Physical Sciences Research Council (EPSRC). Dr Rolf Erni and Ms. Debora Keller from the Electron Microscopy Center, Swiss Federal Laboratories for Materials Science and Technology, are gratefully acknowledged for a very fruitful collaboration on $\mathrm{Cu}(\mathrm{InGa}) \mathrm{Se}_{2}$ absorber layers which lead to some of the results presented here. PZE acknowledges support from the Laboratory Directed Research and Development Program at Pacific Northwest National Laboratory. WPH is supported by the US Department of Energy, Office of Science, Office of Basic Energy Sciences, Division of Chemical Sciences, Geosciences \& Biosciences.
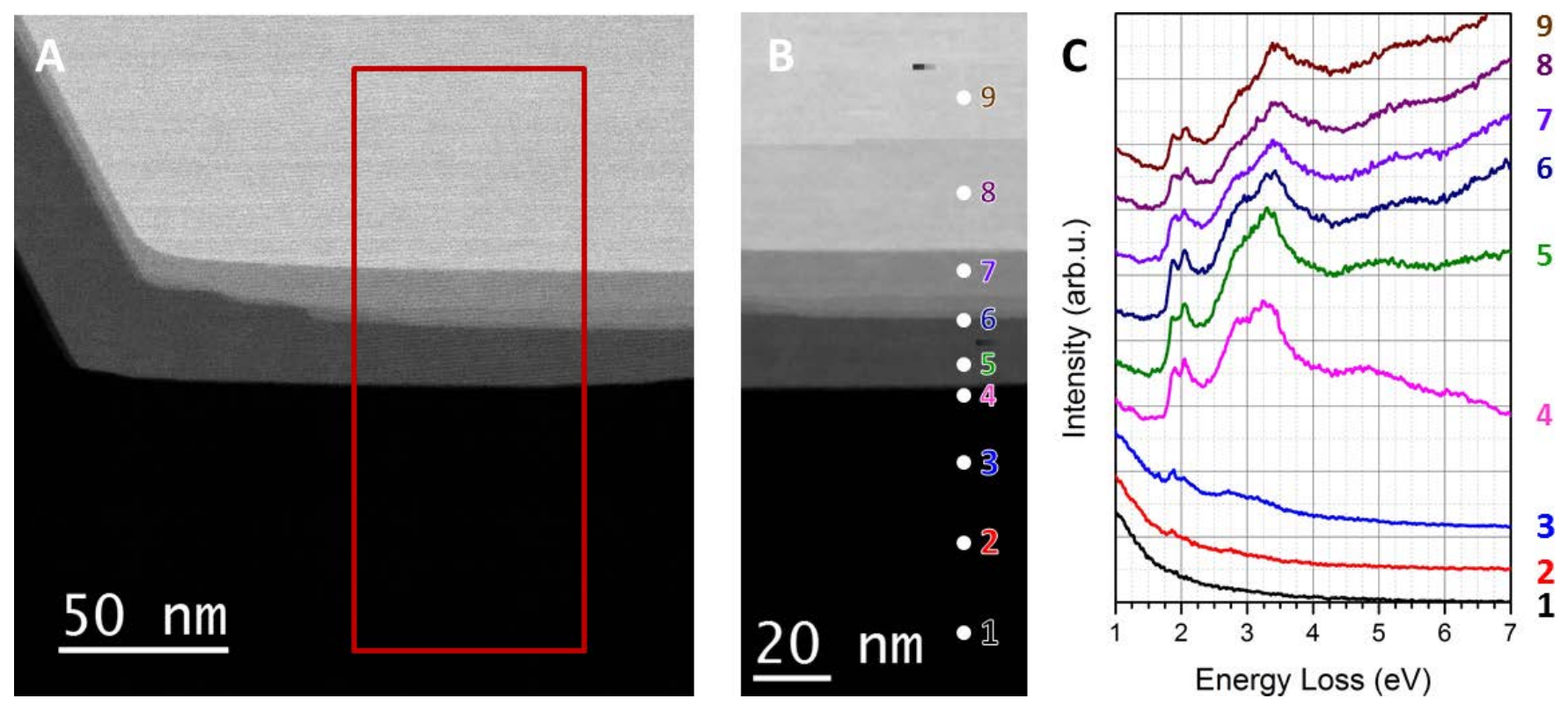

Figure 1. Low loss spectra of a $\mathrm{MoS}_{2}$ nano-flake. The spectra were acquired at positions across step edges and terraces on a mechanically exfoliated nano-flake, as indicated on the survey image, A. The spectra were averaged horizontally at position marked on the simultaneously acquired HAADF image B to provide a higher signal-to-noise. The peaks at $1.9 \mathrm{eV}, \mathrm{C}$, whose splitting is only resolvable thanks to an energy resolution of $\sim 16 \mathrm{meV}$, are shown to be of an excitonic nature and exhibit a clear variation as a function of probe position. Data acquired at $60 \mathrm{kV}$ acceleration voltage on a Nion UltraSTEM100 MC, configured to form a $1.5 \AA ̊$ probe, with $\sim 10 \mathrm{pA}$ of beam current once monochromated to $16 \mathrm{meV}$ energy resolution. 